\title{
2D and 3D Electron Holography Revealing Complex Magnetic Configurations in CoNi Nanowires
}

Ingrid Marie Andersen ${ }^{1}$, Luis Alfredo Rodriguez ${ }^{2}$, Daniel Wolf ${ }^{3}$, Axel Lubk ${ }^{3}$, Cristina Bran ${ }^{4}$, Christophe Gatel $^{1}$ and Etienne Snoeck ${ }^{1}$

${ }^{1}$ CEMES - CNRS, Toulouse, Midi-Pyrenees, France, ${ }^{2}$ Universidad del Valle, Cali, Valle del Cauca, Colombia, ${ }^{3}$ IFW Dresden, Dresden, Sachsen, Germany, ${ }^{4}$ ICMM - CSIC, Madrid, Madrid, Spain

Ferromagnetic nanostructures are considered to be the building blocks for the development of future spintronic devices ${ }^{1}$ where cylindrical nanowires are interesting candidates, particularly as their domain wall propagation has been predicted to surpass the speed which is usually limited by the Walker breakdown in structures like flat nanostrips and thin films. ${ }^{2}$ Understanding the fine structure of domain walls in ferromagnetic nanowires is therefore mandatory. Such difficult studies require a method that allows a quantitative magnetic mapping with nanometer spatial resolution, as well as a three-dimensional representation of magnetic domain walls for cylindrical nanowires. ${ }^{3}$

Electron holography $(\mathrm{EH})$ is a TEM-based technique that offers a spatial resolution down to $0.5 \mathrm{~nm}$ for magnetic imaging. However, only components of the magnetic induction perpendicular to the electron beam path can be measured. ${ }^{4}$ Vector Field Electron Tomography (VFET), however, allows reconstruction of the vector field with all three components of the magnetic induction $\boldsymbol{B}$, giving direct 3D information of the domain wall structure. 5

Through a combined study using EH and VFET at the state of the art, I will present the first quantitative analysis of complex magnetic configurations and domain wall structures in cylindrical CoNi nanowires which display an hcp crystal structure with the c-axis oriented perpendicular to the nanowire axis. The nanowires were studied at a remanent state in two cases after the application of an external saturation field perpendicular (1) and parallel (2) to the nanowire axis. Micromagnetic simulations have also been carried out to complement the experimental results and to give further information about the magnetic characteristics of the sample nanowire.

EH reveals two distinctly different magnetic configurations depending on the two saturation field directions, further characterized by VFET; an unexpected and complex chain of alternating magnetic domains oriented perpendicular to the NW axis for field applied perpendicular to the NW axis, and a curling magnetic configuration oriented axially with respect to the nanowire when the field is applied parallel to the axis. These two magnetic configurations are linked to the strong magnetocrystalline anisotropy of hcp crystal structure, which allows formation of a chain of transversal domain-like magnetic states after the application of an external field directed perpendicular to the nanowire axis, while a curling magnetic state is the preferred remanent state when the external field has been applied to align the magnetic spins along the nanowire axis. In the latter case, after the removal of the applied field, the strong c-axis anisotropy realigns the magnetic configuration to create this curling configuration, rather than a completely axially saturated state. These configurations are confirmed by micromagnetic simulations. 
a)

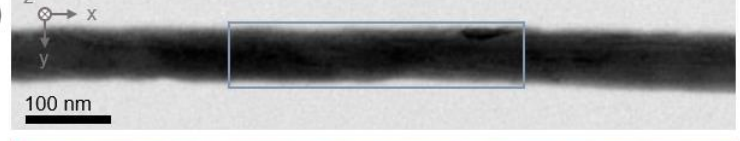

b)

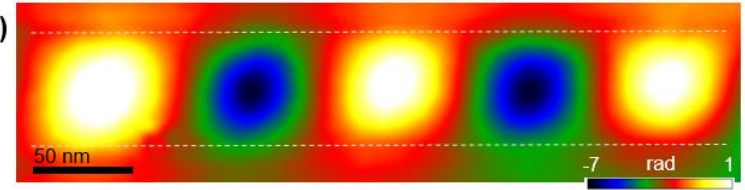

c)

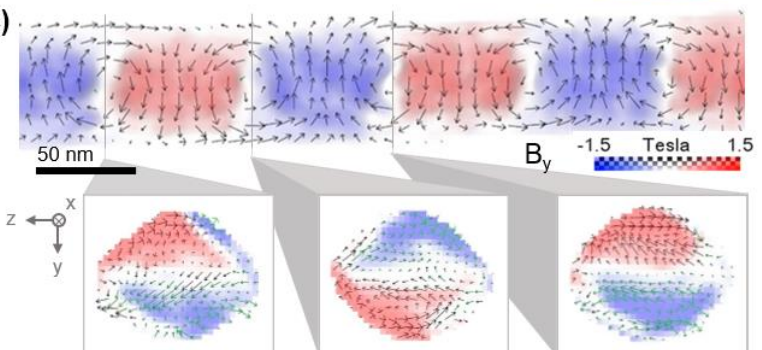

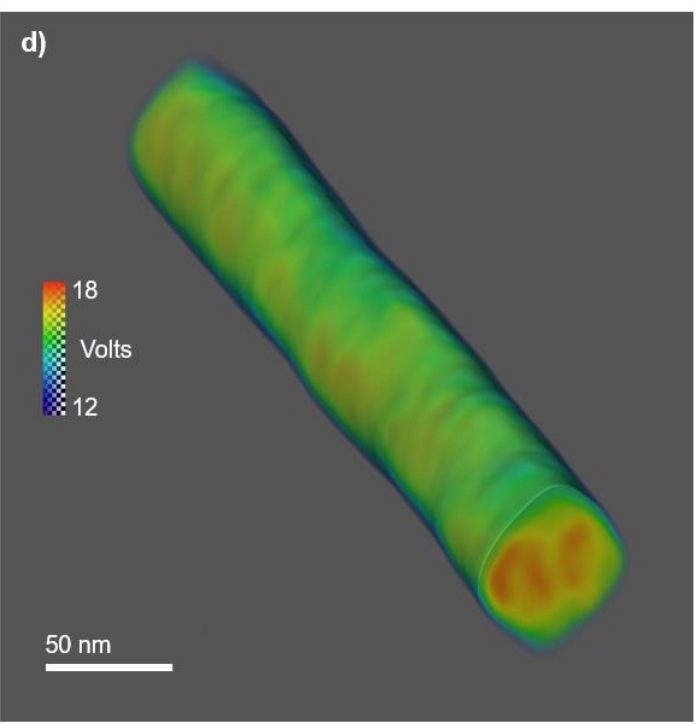

Figure 1. (a) BF-TEM image of studied CoNi nanowire. (b) Magnetic phase image from electron holography of boxed region in (a) recorded at remanent state after an applied saturation field oriented perpendicular to the nanowire axis showing a chain of vortex-like domains. (c) Central axial slice through the 3D vector-field of the magnetic induction $\boldsymbol{B}$ reconstructed by holographic vector-field electron tomography, and three cross-sections at selected vortex cores (positions marked by lines). (d) 3D electrostatic potential reconstructed by holographic tomography visualizing the 3D morphology of the NW.

\section{References}

1. Hertel, R. Ultrafast Domain Wall Dynamics in Magnetic Nanotubes and Nanowires. J. Phys. Condens. Matter 2016, 28, 483002.

2. Berger, L. Emission of Spin Waves by a Magnetic Multilayer Traversed by a Current. Phys. Rev. B 1996, 54, 9353-9358.

3. Da Col, S.; Jamet, S.; Rougemaille, N.; Locatelli, A.; Mentes, T. O.; Burgos, B. S.; Afid, R.; Darques, M.; Cagnon, L.; Toussaint, J. C.; et al. Observation of Bloch-Point Domain Walls in Cylindrical Magnetic Nanowires. Phys. Rev. B 2014, 89, 180405.

4. Lichte, H.; Lehmann, M. Electron Holography_Basics and Applications. Rep. Prog. Phys. 2008, 71, 016102.

5. Wolf, D.; Biziere, N.; Sturm, S.; Reyes, D.; Wade, T.; Niermann, T.; Krehl, J.; Warot-Fonrose, B.; Büchner, B.; Snoeck, E.; et al. Holographic Vector Field Electron Tomography of Three-Dimensional Nanomagnets. Commun. Phys. 2019, 2, 1-9. 